\title{
Strategies for the management of hypertension in aircrew
}

\author{
H. R. BRUNNER, B. WAEBER AND J. NUSSBERGER
}

Hypertension Division and Cardiovascular Research Group, University Hospital, Lausanne, Switzerland

KEY WORDS: Aircrew licensing, aviation, hypertension, management, treatment.

\begin{abstract}
Introduction
Agencies responsible for the regulation of civil aviation are required to contain the risk of crew incapacitation. Consequently, strategies to identify and exclude pilots presenting with an excess high risk have been evolved. Disabling cardiovascular events such as stroke or myocardial infarction, as well as arrhythmias and sudden cardiac death, represent the main cardiovascular causes of sudden inflight incapacity to fly an airplane. The concept of risk with particular reference to aircrew and the probability of cardiovascular event has already been discussed extensively ${ }^{[1-4]}$. It became evident in these analyses that age and gender represent risk factors of unique importance ${ }^{[5]}$. Below retirement age, women have a lower risk of mortality from ischaemic heart disease. Furthermore, particularly in men, mortality rates from ischaemic heart disease increase steeply above the age of 45-50. Nothing is likely to change these two variables, although mortality in aircrew has been found to be lower than expected from epidemiological studies in the general population ${ }^{[4]}$. These findings are not altogether surprising, as pilots represent a selected population characterized by motivation towards good cardiovascular health.

How does hypertension affect this risk, and what can be
\end{abstract} done about it? There exists overwhelming evidence that hypertension increases the risk of myocardial infarction and stroke $e^{[0-8]}$. There is also good evidence that stroke can be prevented by antihypertensive therapy $y^{[9]}$. The incidence of stroke is, however, much less than that of myocardial infarction. Therefore, although the prevention of myocardial infarction ${ }^{[8]}$ by antihypertensive therapy should provide a greater benefit, unfortunately, the evidence that this can be achieved effectively is less convincing. Indeed, very few controlled prospective antihypertensive intervention trials show clear evidence of prevention of myocardial infarction. Only meta-analysis of such studies with the inherent shortcomings of the technique have been able to provide some evidence suggesting a significant, albeit much lower than expected decrease in the incidence of myocardial infarction ${ }^{\text {99] }}$. It seems thus unlikely that antihypertensive treatment, per se, can make much contribution to the safety of air travel.

The risk of suffering a heart attack even in a European male is very low below the age of $50^{(5)}$, thus screening for predictors of such an event can only be useful if such

Correspondence: Hans R. Brunner, MD, Hypertension Division, CHUV, 1011 Lausanne, Switzerland. variables have a high degree of specificity. Electrocardiographic screening of symptom-free subjects identifies a very small percentage of pilots at high risk but also misses others whose risk is not normal for their age. The previous occurrence of myocardial infarction and/or a history of angina pectoris does, however, identify subjects at a markedly higher risk ${ }^{[3]}$ who will be excluded from flying a commercial aircraft.

But pilots with an elevated blood pressure are also patients. The main goal of therapy thus should be to reduce the blood pressure and hopefully prolong the life expectation of the individual pilot, even though this will have little or no impact on flight safety overall. Of importance, antihypertensive therapy should be appropriate to the operational environment. No new risk should be introduced by the therapeutic agent which may potentially hamper the flying capacity of the pilot through such unwanted effects as orthostatic hypotension, arrhythmia or sedation.

In the following text, a management strategy is proposed for aircrew who upon screening exhibit a consistently elevated blood pressure.

\section{Elevated blood pressure in aircrew}

The first requirement is to decide whether a subject has 'true' hypertension, or whether his/her blood pressure is elevated only during a visit to the doctor - what commonly we term 'white coat' hypertension. There is some indication, though not conclusive evidence, that the blood pressure taken in the physician's office is a less good predictor of risk than the blood pressure measured while the patient pursues his usual activities ${ }^{[10-12]}$. Approaches used to enhance the accuracy of blood pressure evaluation are self-measurement by the patient, or ambulatory blood pressure recording, discussed elsewhere in this issue. In our laboratory, any blood pressure measured outside the clinic that is on daily average higher than $140 / 90 \mathrm{mmHg}$ is considered abnormal and in need of therapeutic care.

When the presence of a high blood pressure is established, the usual work-up begins with a full history. Particular care is taken to identify indicators of cardiovascular disease, such as previous events, angina pectoris, dyspnoea or intermittent claudication ${ }^{[13]}$. The physical examination should be conducted according to accepted practices $^{[14]}$. A search should be made for the presence of peripheral vascular disease by examination of the optic 
fundus, auscultation for arterial bruits and palpation of peripheral pulses. The presence or absence of a fourth heart sound should be noted. The laboratory work-up should include an electrocardiogram, a blood count and a complete urine analysis. Measurements should also be taken of plasma glucose, potassium, creatinine, uric acid, total cholesterol, HDL cholesterol and triglycerides.

Additional investigations may be justified to characterize abnormal findings. These complementary tests may serve to clear a suspicion of abnormality and in a few they will confirm the existence of a problem which may be of concern from the certificatory point of view.

\section{Treatment}

\section{NON-PHARMACOLOGICAL THERAPY}

The non-pharmacological approach to the treatment of hypertension has the appeal of introducing few untoward effects. The efficacy of some of these interventions is, however, still debated. All such therapies require a continued effort by the patient, and therefore the critical issue is, whether long-term blood pressure control can be achieved and maintained by these means. Most of the trials have studied the different intervention procedures only over a relatively short period in order to prove their efficacy. Over a longer period of time, results are often more disappointing. For this reason, non-pharmacological antihypertensive therapy should probably be regarded in most cases as a supportive measure rather than an alternative approach to drug treatment.

\section{WEIGHT REDUCTION}

The different non-pharmacological approaches to the treatment of hypertension in aircrew have been reviewed previously in some detail ${ }^{[15]}$. Among the dietary measures, weight reduction by reduction in calorie intake seems today quite well established as an effective means of reducing blood pressure. While early uncontrolled studies demonstrated blood pressure reduction when grossly overweight patients lost $10 \mathrm{~kg}$ or more of weight ${ }^{[16.17]}$, more recent studies have reported blood pressure reduction with weight loss in the vicinity 4 to $5 \mathrm{~kg}^{[18,19]}$. In a recent study using ambulatory blood pressure monitoring, we found a blood pressure reduction which was clearly related to the actual weight loss ${ }^{[20]}$. Furthermore, those patients who lost weight, not only reduced their blood pressure but also reduced their free cytosolic platelet calcium. However, results after 1 to 2 years were disappointing since most of the patients included were back to their original weight. Thus, even if blood pressure can be reduced by weight reduction, long-term maintenance by this therapy seems unreliable ${ }^{[21]}$.

\section{REDUCTION OF SODIUM INTAKE}

Reduction of sodium intake appears rational since there exists plenty of circumstantial experimental and clinical evidence to suggest that sodium retention may play an important role in the pathogenesis of hypertension ${ }^{[22]}$. There are also some very well conducted trials which have demonstrated that blood pressure can be lowered by a reduction in sodium intake $\mathrm{e}^{[23-25]}$. The problem is that the impact of moderate salt restriction on blood pressure is relatively modest. The same can be said for potassium supplementation ${ }^{[26}$. Moderate salt restriction tends to enhance the antihypertensive efficacy of some pharmacological agents and this seems sensible as a supportive measure in the management of hypertension.

\section{VEGETARIAN DIET}

It is likely that vegetarian diets also reduce the ratio of the dietary sodium/potassium intake. In addition they decrease the intake of animal lipids. This may explain why, in a well controlled study, a vegetarian diet was effective in reducing blood pressure ${ }^{2 \pi}$. Although of interest from an investigational standpoint, this observation is clinically probably not very useful. Calcium supplementation, advocated by some, has not proven its usefulness in the treatment of hypertension ${ }^{[28]}$, although the reduction of consumption or withdrawal is of accepted benefit.

\section{EXERCISE}

With regard to life style change, regular exercise can reduce the blood pressure ${ }^{[29]}$. Notwithstanding, many patients will not maintain a regular and relatively intensive exercise programme over a period of many years. Accordingly, there are probably few hypertensive patients who can be successfully treated for years by regular exercise alone. Biofeedback and relaxation therapy are more difficult to assess since it is almost impossible to rule out the placebo effect of the measure.

Taken together, non-pharmacological approaches to the treatment of hypertension are reasonable, supportive measures since they represent in general a healthier life style. While they can be considered risk-free, when applied excessively they may not be completely harmless. In particular, low sodium intake in combination with somedrugs may trigger hypotension and excessive potassium or calcium supplementation could be ill-advised in patients with reduced renal function. Furthermore, over-emphasis of these measures could lead in some patients, who cannot meet the requirements, to unnecessary feelings of guilt which may interfere with their quality of life.

\section{PHARMACOLOGICAL THERAPY}

There are many different classes of drugs available for the treatment of hypertension. For all practical purposes, these drugs can be considered to exhibit similar antihypertensive efficacy, even though this may vary from one class to another in the individual patient. Accordingly, in this general discussion concerning the usefulness of different classes of drug, efficacy is not an issue. Efficacy becomes important when trying to match the right drug to the right patient.

One of the main considerations when choosing an antihypertensive drug for a pilot must be attention to the side-effect profile of the agent used. Side-effects that are unacceptable include hypotension, arrhythmia, depression and drowsiness. Consideration also has to be given to drug interactions, since drug combinations are

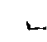

$$
\text { . }
$$


often necessary to normalize blood pressure. These interactions may be detrimental or beneficial; thus side-effects may only occur when two drugs are administered together or the side-effects of a certain drug may be attenuated by the co-administration of a second agent.

There are some other considerations to be made when administering an antihypertensive drug. Most of the antihypertensive drugs have been given in the higher recommended dose, which in some instances has undoubtedly led to an unnecessary high incidence of side-effects. Furthermore, the treating physician has to choose between a strategy of early combination of two or more antihypertensive drugs or, alternatively, the sequential trial of the various classes of antihypertensive drugs. These concepts have been discussed elsewher $\mathrm{e}^{[30]}$.

In the treatment of aircrew, sequential monotherapy may be preferable to obtain a clear profile of potential side-effects. Whatever strategies are chosen, monotherapy or a combination of agents, any drug should be used whenever possible at the lower limit of the recommended dosage range.

\section{AGENTS TO AVOID}

Before discussing the drugs to be recommended for the treatment of aircrew, it is useful to identify those drug classes that should not be used. First, there are the centrally acting drugs, such as reserpine, clonidine, guanabenz, guanfacine and methyldopa, and possibly the lipophilic beta-blocking agents. It is not easy to provide evidence that task performance may be impaired by these drugs $^{[3 !]}$. Notwithstanding, better agents exist. A second class of drugs that probably should be avoided includes the alpha-adrenoceptor blocking agents, such as phenoxybenzamine, phentolamine, prazosin, trimazosin, doxazosin, terazosin and urapidil. This group may provoke some degree of orthostatic hypotension, particularly after the first dose $\mathrm{e}^{[32]}$. Some of the newer agents are claimed to cause little such hypotension, but it appears preferable to avoid this class of drugs in the treatment of aircrew.

\section{PERMISSIBLE AGENTS}

The remaining 'permissible' classes of agents include the diuretics, the beta-adrenoceptor blocking agents, the calcium antagonists and the angiotensin II converting enzyme (ACE) inhibitors, which all can be used as first step therapy, and the vasodilators, which are recommended mainly in association with the other drugs.

\section{Diuretics}

The diuretic agents represent the longest established class that is currently still in general use for the treatment of hypertension. Among the diuretics, hydrochlorothiazide and the various thiazide derivatives are probably preferable as they induce a smooth and progressive sodium loss ${ }^{[33]}$. Side-effects such as potassium loss, hyperuricaemia and relative glucose intolerance are all of gradual onset and are unlikely to cause any sudden in-flight incapacitation. Potassium sparing diuretics such as spironolactone, amiloride and triamterene are equally useful, be it alone or in combination with thiazide diuretics, and they provide the advantage of protecting against hypokalaemia. Doses should not exceed $25 \mathrm{mg}$ od of hydrochlorothiazide or the equivalent for the thiazide derivatives or 50-75 mg od spironolactone. Loop diuretics are rarely necessary, unless there exists some degree of renal failure and should be avoided altogether in the treatment of aircrew because of the potential risk of excessive salt deprivation which could lead to hypotension. Urinary frequency is also likely to be a problem. Diuretics can be combined with any of the three classes, i.e. beta-blockers, calcium antagonists and ACE inhibitors, with good additive antihypertensive efficacy, though the increase in efficacy when combined with calcium antagonists is debated $^{[34]}$. In combination with ACE inhibitors, diuretic induced hypokalaemia, hyperuricaemia and possibly also glucose and lipid alterations may be attentuated ${ }^{[35,36]}$.

\section{Betablockers}

Beta-adrenoceptor blocking agents have been considered the gold standard for aircrew treatment ${ }^{[37]}$. As already mentioned on theoretical grounds preference should be given to compounds with a low lipophilicity, such as atenolol, nadolol and sotalol ${ }^{[38]}$. It should, however, not be forgotten that beta-blockers can cause sideeffects such as fatigue, drowsiness, asthma, Raynaud's phenomenon, and impairment of lower limb circulation. Beta-blockers may be used with any drug of the three other classes, though the additive efficacy in combination with ACE inhibitors may become evident only in a relatively limited fraction of patients. Beta-blockers can be safely combined with calcium antagonists of dihydropyridine type, but association with verapamil or diltiazem should be avoided because of the risk of inducing atrio-ventricular block.

\section{Calcium antagonists}

The slow channel calcium blocking agents and the ACE inhibitors represent newer classes of drugs. Because of lack of long-term experience, they have not been recommended in the past for treatment of flight crew ${ }^{[37]}$. Even today, there is still relatively little evidence available demonstrating the beneficial effects of these compounds in hypertensive patients, though their antihypertensive efficacy is well established. By now, both drug classes have, however, proven to be well tolerated and safe, and for this reason they should be good candidates for use in the treatment of hypertensive pilots. In particular, they do not seem to exhibit significant central effects and they should not induce orthostatic hypotension.

The calcium antagonists form probably the least homogenous group since diltiazem and verapamil are quite different from the dihydropyridine derivatives ${ }^{[39]}$, and even among the latter there exist considerable differences in the side-effect profile ${ }^{[40]}$. Side effects such as flushing, headache and oedema are common but even so, when used as monotherapy, probably any of the calcium antagonists could be considered safe for the treatment of hypertensive pilots. Preference may be given to the longer acting drugs in order to avoid side-effects such as palpitation related to rather more abrupt onset of action. Apart from 
verapamil they are probably best combined with a betablocking agent and dihydropyridine derivatives are best used in this context as already outlined above.

\section{$A C E$ inhibitors}

The ACE inhibitors also do not cause orthostatic hypotension in the sodium replete individual, and do not impair cardiac function ${ }^{[4]]}$. In combination with diuretic therapy, they are particularly effective but loop diuretics should be avoided. Preference here also should be given to longer acting agents using the lower recommended dose, e.g. lisinopril or enalapril at a dose of $10 \mathrm{mg}$ od or equivalent. Side-effects such as cough or angioneurotic oedema should not occur sufficiently suddenly to incapacitate a pilot during the average duration of a flight.

The directly acting vasodilators such as hydralazine, dihydralazine or minoxidil are not recommended as monotherapy ${ }^{[42]}$. They may, however, be used beneficially in combination with a beta-blocker or possibly an ACE inhibitors. Today, most often calcium antagonists have taken their place. The use of minoxidil in the treatment of aircrew should be avoided because of the risk of hypotension.

\section{Conclusions}

The four differing classes of antihypertensive drugs, i.e. the diuretic agents, beta-adrenoceptor blocking agents, slow channel calcium blockers and ACE inhibitors, provide a good array of therapeutic tools with which to treat hypertensive pilots. The use of low doses in monotherapy as well as in combination is an important feature in any strategy. Sequential monotherapy, that is a sequential trial of a representative of each of these four classes, in the hope of identifying one compound able to control blood pressure when given alone, is to be recommended. Combination therapy may be used, if no single agent provides good blood pressure control. Diltiazem and verapamil should not be combined with beta-blocking agents, and loop diuretics should be avoided altogether, particularly in combination with ACE inhibitors.

Because most pilots belong to an age group which exhibits a low incidence of sudden incapacitating cardiovascular complications due to hypertension, antihypertensive therapy, a priori, has only a small potential to exert a beneficial effect on air safety. The screening of aircrew should identify predictors of high risk for an incapacitating cardiovascular event, including co-existing lipid disorder, smoking and family history of premature cardiovascular disease. Antihypertensive strategy in aircrew is similar to that used for the general population and the long-term objectives are the same - the maintenance of optimum long-term health in a pharmacological environment as free from unwanted effects as possible.

\section{References}

[1] Tunstall-Pedoe H. The concept of risk. Eur Heart J 1988; 9 (Suppl G): 13-15.
[2] Chaplin JC. In perspective - the safety of aircraft, pilots and their hearts. Eur Heart J 1988; (Suppl G); 17-20.

[3] Pocock SJ, Shaper AG, Phillips AN, Walker M. Prediction of men at high risk of heart attack and its relevance to pilots. Eur Heart J 1988; 9 (Suppl G): 25-30.

[4] Tyler A. How actuaries and underwriters look at risk. Eur Heart J 1988; 9 (Suppl G): 31-35.

[5] Tunstall-Pedoe $H$. Introduction to section I. Acceptable cardiovascular risk in aircrew. Eur Heart J 1988; 9 (Suppl G): 9-11.

[6] Lew EA. High blood pressure, other risk factors and longevity: the insurance viewpoint. Am J Med 1973; 55: 281-94.

[7] Kannel WB. Role of blood pressure in cardiovascular morbidity and mortality. Progr Cardiovase Dis 1974; 17: 5-23.

[8] MacMahon S, Peto R, Cutler J, et al. Blood pressure, stroke, and coronary heart disease. 1. Prolonged differences in blood pressure: prospective observational studies corrected for the regression dilution bias. Lancet 1990; i. 765-74.

[9] Collins R, Peto R, MacMahon S, et al. Blood pressure, stroke, and coronary heart disease. 2. Short-term reductions in blood pressure: overview of randomised drug trials in their epidemiological context. Lancet 1990; i: 827-38.

[10] Perloff D, Sokolow M, Cowan R. The prognostic value of ambulatory blood pressure. JAMA 1983; 249; 2792-8.

[11] Perloff D, Sokolow M, Cowan RM, Juster RP. Prognostic value of ambulatory blood pressure measurements: further analysis. J Hypertens 1989; 7 (Suppl 3): 3-10.

[12] Pickering TG, Harshfield GA, Devereux RB, Laragh JH. What is the role of ambulatory blood pressure monitoning in the management of hypertensive patients. Hypertension 1985; 7: $171-7$

[13] Ledingham JGG. Peripheral vascular disease as a risk factor for ischaemic heart disease. Eur Heart J 1988; 9 (Suppl G): 65-8.

[14] Muller FB, Laragh JH. Clinical evaluation and differential diagnosis of the individual hypertensive patient. In: Laragh JH, Brenner BM, eds. Hypertension: Pathophysiology, Diagnosis, and Management. New York: Raven Press, Ltd, 1990: 1385-96.

[15] Swales JD. Non-pharmacological antihypertensive therapy. Eur Heart J 1988; 9 (Suppl G): 45-52.

[16] Heyden S, Tyroler A, Hames CG et al. Diet treatment of obese hypertensives. Clin Sci Mol Med 1973; 45: 294-325.

[17] Schlundt DG, Langford HG, McDonel EC. Compliance in dietary management of hypertension. Compr Ther 1985; 11 : 59-66.

[18] Reisin E, Rachel A, Modan M, Silverberg DS, Eliahou HE, Modan B. Effect of weight loss without salt restriction on the reduction of blood pressure in overweight hypertensive patients. N Engl J Med 1978; 298: 1-6.

[19] Stamler R, Stamler J, Grimm R et al. Nutritional therapy for high blood pressure. Final report of a four-year randomised controlled trial. The hypertension control program. JAMA 1987; 257: 1484-91.

[20] Scherrer U, Nussberger J, Torriani S et al. Effect of weight reduction in moderately overweight patients on recorded ambulatory blood pressure and free cytosolic platelet calcium. Circulation 1991; 33: 552-8.

[21] Dunbar J. Practical aspects of dietary management of hypertension: compliance. Can J Physiol Pharmacol 1986; 64: 831-35.

[22] Weinberger $\mathrm{MH}$. Clinical studies of the role of dietary sodium in blood pressure. In: Laragh JH, Brenner BM, eds. Hypertension: Pathophysiology, Diagnosis, and Management. New York: Raven Press, Ltd. 1990: 1999-2010.

[23] Parijs J, Jossens JV, Van der Linden L, Verstreken G, Amery $A K P C$. Moderate sodium restriction and diuresis in the treatment of hypertension. Am Heart J 1973; 85: 22-34.

[24] MacGregor GA, Markandn N, Best F, Elder D, Cam J, Squires $M$. Double-blind randomised crossover trial of moderate sodium restriction in essential hypertension. Lancet 1982; $\mathrm{i}$ : $351-4$.

[25] Morgan T, Gillies A, Morgan G, Adam W, Wilson M, Carney S. Hypertension treated by salt restriction. Lancet 1978 ; $\mathrm{i}$ : 227-33. 
[26] Iimura O, Kijima K, Miyama A, Ando T, Nakao T, Takigami $Y$. Studies on the hypotensive effect of high potassium intake in patients with essential hypertension. Clin Sci 1981; 61: 77s-80s.

[27] Margetts BM, Beilin LJ, Vandongen R, Armstrong BK Vegetarian diet in mild hypertension: a randomised controlled trial. Br Med J 1986; 293: 1468-71.

[28] Kaplan NM, Meese RB. The calcium deficiency hypothesis of hypertension: a critique. Ann Int Med 1986; 105: 947-58.

[29] Nelson L, Jennings GL, Esler MD, Korner PI. Effects of changing levels of physical activity on blood pressure and haemodynamics in essential hypertension. Lancet 1986; ii: 473-76.

[30] Brunner HR, Menard J, Waeber B et al. Treating the individual hypertensive patient: Considerations on dose, sequential monotherapy and fixed-dose combinations. J Hypertens 1900 ; 8: 3-11.

[31] Callender JS. Evaluation of the cerebral effects of antihypertensive medication Eur Heart J 1988; 9 (Suppl G): 59-63.

[32] Van Zwieten PA. Alpha-adrenoceptor-blocking agents in the treatment of hypertension. In: Laragh JH, Brenner BM, eds. Hypertension; Pathophysiology, Diagnosis, and Management. New York: Raven Press, Ltd 1990: 2233-49.

[33] Pecker MS. Pasthophysiologic effects and strategies for longterm diuretic treatment of hypertension. In: Laragh JH, Brenner, BM eds. Hypertension: Pathophysiology, Diagnosis, and Management. New York: Raven Press, Ltd, 1990: 2143-67.

[34] Nicholson JP, Resnick LM, Laragh JH. Hydrochlorothiazide is not additıve to verapamil in treating essential hypertension. Arch Int Med 1987; 107: 150-7.
[35] Weinberger $\mathrm{MH}$. Infuence of an angiotensin-converting enzyme inhibitor on diuretic-induced metabolic effects in hypertension. Hypertension 1983; 5 (Suppl III): 132-8.

[36] Vidt DG. A controlled multiclinic study to compare the antihypertensive effects of MK-421, hydrochlorothiazide, and MK421 combined with hydrochlorothiazide in patients with mild to moderated essential hypertension. J Hypertens 1984; 2 (Suppl 2): 81-8.

[37] Ball SG. New anti-hypertensive agents and their use by aircrew, Eur Heart J 1988; 9 (Suppl G): 53-7.

[38] Bolli P, Fernandez PG, Buhler FR. Beta-blockers in the treatment of hypertension. In: Laragh JH, Brenner BM, eds. Hypertension: Pathophysiology, Diagnosis, and Management. New York: Raven Press, Ltd, 1990: 2181-208.

[39] Henry PD. Comparative pharmacology of calcium anatag. onists; nifedipıne, verapamil and diltiazem. Am J Cardiol 1980; 46: $1047-58$

[40] Freedman DD, Waters DD. 'Second generation' dihydropyridine calcium antagonists. Greater vascular selectivity and some unique applications. Drugs 1987; 34: 578-98.

[41] Waeber B, Nussberger J, Brunner HR Angiotensin-convertingenzyme inhibitors in hypertension. In: Laragh JH, Brenner BM, eds. Hypertension; Pathophysiology, Diagnosis, and Management. New York: Raven Press, Ltd, 1990: 2209-32.

[42] Robinson BF, Benjamin N. Vasodilators . In: Laragh JH, Brenner BM, eds. Hypertension: Pathophysiology, Diagnosis, and Management. New York: Raven Press, Ltd, 1990: 2263-74. 